\title{
IMPROVING THERMOMECHANICAL PROPERTIES OF FUSED FILAMENT FABRICATION PRINTED PARTS BY USING NANOCOMPOSITES
}

\author{
GEOFFREY GINOUX, PHILIPPE DONY, ISABELLE VROMAN, SEBASTIEN ALIX
}

Institut de Thermique, Mécanique, Matériaux - Université de Reims Champagne-Ardenne, 51100 Reims (France) - Email: geoffrey.ginoux@univ-reims.fr

\begin{abstract}
.
This study aims to assess the viability of nanocomposites in fused filament fabrication. Polylactide/organo-modified layered silicate materials have been studied in terms of structure and properties evolution through the whole manufacturing chain, and for thermomechanical optimisation. The method included the optimisation by the composite formulation and by the additive manufacturing process by experimental designs. A competition between flow thinning of the molten composite and stiffness of the input filament with the addition of filler allows an enhanced printability at intermediate loadings. The method provides an optimisation of the final parts, enabling similar or greater tensile properties, while providing better thermal stability, with additively manufactured nanocomposite materials compared to conventionally manufactured polymers.
\end{abstract}

Keywords: A. Polymer-matrix composites (PMCs), B. Microstructures, B. Thermomechanical, C. Statistical properties/methods, Additive manufacturing.

\section{Introduction}

Initially used for rapid prototyping, additive manufacturing (AM) processes offer the possibility to manufacture complex parts thanks to their layer-by-layer assembly, which enables the fabrication of expensive end-use products at lower cost, or of impossible-to-manufacture parts with usual subtractive or formative techniques. However, a loss in mechanical performance is observed with additively manufactured parts compared to those produced by subtractive and formative processes, which prevents the development of this technology towards small-series production or medical applications [1]. This development is a major issue and requires new materials to design fully functional products [2]. The addition of fillers in a polymer matrix is one way to adapt the rheological and functional properties, and to expand their potential uses [3]. Fused Filament Fabrication (FFF) technique is one of the most commonly AM process used due to its speed and costeffectiveness [4], but the use of micrometric fillers could cause the nozzle to clog because of the filler dimensions compared to the low nozzle diameter [5]. Nanofillers appear as an obvious solution to get around this geometric problem. One particular nanofiller is the organo-modified layered silicate (OMLS), a clay (phyllosilicate) in the form of nanoplatelets of around $1 \mathrm{~nm}$ in thickness and 100 to $1000 \mathrm{~nm}$ in width and length. These dimensions develop a high specific surface area of around $800 \mathrm{~m}^{2} / \mathrm{g}$, which allows the properties development at low loadings, less than $10 \mathrm{wt} \%$ against 40 to $60 \mathrm{wt} \%$ for microfillers [6], which would again decrease the nozzle clogging risk. Ray et al. [7] revealed several property improvements thanks to OMLS, from thermal stability and gas barrier properties, to mechanical reinforcement. Consequently, the use of nanocomposites is one promising solution to move the FFF technology towards small-series productions of functional end-use products [8]. With the use of polylactide (PLA) as the matrix, a bio-sourced, biodegradable and biocompatible aliphatic polyester, the applications are expected, but not limited, in aerospace, medical field or packaging [9].

Independently of the kind of fillers, a thickening effect is observed with the addition of particles in a molten polymer [10], which could prevent the materials to flow through the nozzle. The printability of nanocomposite systems is then not certain. An optimisation of the OMLS loadings is expected with a competition between the ability to be shaped by FFF and the development of thermomechanical properties.

The aim of this article is to assess the viability of additively manufactured PLA/OMLS-based materials while simultaneously reaching similar or greater thermomechanical performance than formative neat PLA one. Indeed, it is wellknown that printed parts generally have lower mechanical properties compared to injected parts, and therefore have lower thermodynamic properties. Thermomechanical performance in this study includes thermal stability and tensile properties, namely Young's modulus, ultimate tensile strength and elongation at break. PLA/OMLS-based nanocomposites silicates are proposed in fused filament fabrication to extent this technology towards the small series production of functional and customisable parts. Targeted applications may include on-demand part production during long-duration space missions where high thermomechanical performance are needed, customisable packaging where barrier properties are sought, or 
medical applications for drug control delivery in bone tissue engineering. As the rheology is dependent on temperature, shear rate and OMLS loading [11], the optimisation must consider the thermokinetic process parameters in addition to the formulation. The viability of nanocomposites includes (1) the possibility of use and (2) the capacity of the technology to freely tailor thermomechanical properties. A method is introduced in this study, including multi-physics characterisation and modelling/statistical tools for performance tailoring. Thermal properties are characterised by thermogravimetric analysis (TGA) to obtain the thermal stability and by differential scanning calorimetry (DSC) to assess the crystallinity rate and the phase transitions. Mechanical properties are characterised by tensile tests and optimised using Doehlert designs, a response surface methodology for properties optimisation and process understanding with a time and cost efficiency [12]. Two factors are considered, namely printing speed and temperature. Flow behaviour is defined by capillary rheology. Formulation state is determined by gel permeation chromatography (GPC) for polymer molar mass, TGA for filler loading, and X-ray diffraction (XRD) and transmission electronic microscopy (TEM) for filler dispersion morphology.

\section{Materials and methods}

The overall experimental section is depicted in the following schematic diagram (see Figure 1).

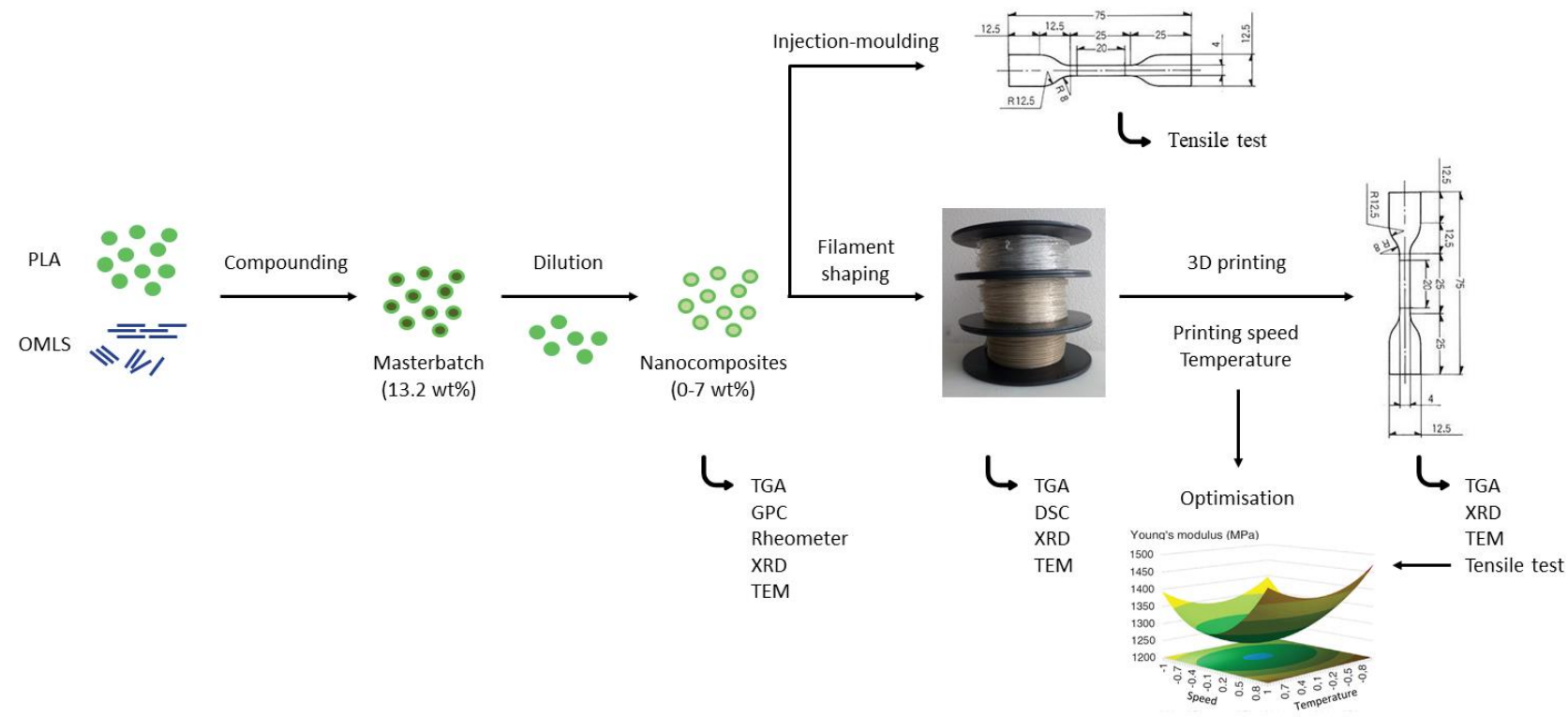

Figure 1. Schematic diagram of the overall experimental section, from materials preparation to characterisation tests.

2.1. Materials and nanocomposite preparation

PLA was purchased from NaturePlast (Ifs, France) in pellets form under the reference PLE 005, an extrusion grade. PLA's specific gravity is 1.26 . The OMLS was an organically modified montmorillonite (MMT) supplied by Southern Clay Products (Gonzales, TX, USA) under the trade name Cloisite ${ }^{\circledR}$ 30B (C30B). The modifier was the methyl, tallow, bis-2hydroxyethyl, and quaternary ammonium with a concentration of $90 \mathrm{mEq}$ per $100 \mathrm{~g}$. C30B's specific gravity is 1.98 , and that of native MMT is 2.86 .

PLA was first dried at $70^{\circ} \mathrm{C}$ overnight before nanocomposite compounding with a co-rotating twin-screw extruder Clextral $\mathrm{BC} 21(\mathrm{D}=25 \mathrm{~mm}, \mathrm{~L} / \mathrm{D}=36)$ with temperatures set between $165{ }^{\circ} \mathrm{C}$ and $180{ }^{\circ} \mathrm{C}$ at $300 \mathrm{rpm}$. The residence time was approximately three minutes with a mass flow of $1.8 \mathrm{~kg} / \mathrm{h}$. A masterbatch at $13.2 \mathrm{wt} \%$ of clay in PLA was obtained. The masterbatch was then dried and diluted with PLA at desired loadings of $0.5,1,3,5$, and $7 \mathrm{wt} \%$. Samples were named PLA05, PLA1, PLA3, PLA5, and PLA7, respectively. PLA also underwent the extrusion process, and the sample was named PLA0. This method permitted us to reach a better nanoparticle dispersion and orientation, as described by Alix $e t$ al. [13] for polyamide nanocomposites, which is essential to develop the composite properties compared to the added amount of clay [14]. Materials were then pelletised using a Scamia granulator $960001 \mathrm{GR}$.

Pellets were first dried overnight at $70{ }^{\circ} \mathrm{C}$ in a ventilated oven and then shaped into a $1.65 \pm 0.05 \mathrm{~mm}$ diameter filament thanks to a single screw extruder Axon BX10 ( $\mathrm{D}=10 \mathrm{~mm}, \mathrm{~L} / \mathrm{D}=18)$ with a screw speed at $20 \%$ of maximum speed and a $0,46 \mathrm{~kg} / \mathrm{h}$ mass flow. The profile temperature was set to $190{ }^{\circ} \mathrm{C}, 185^{\circ} \mathrm{C}, 185^{\circ} \mathrm{C}$ from feeding zone to nozzle. Filaments were cooled with a water bath at room temperature. The filament was pulled by a Haake PP1 to obtain the required diameter. 
Once filament obtained, tensile test specimens were manufactured using a Makerbot Replicator 2 with a 0.4 mm diameter nozzle. The specimens were modelled using Solidworks software with dimensions according to 5A-type specimens from ISO 527-2 standard. STL file was then imported to the Makerbot Print software. Specimens were printed with two shells, in a $90^{\circ} / 0^{\circ}$ crossed-deposition fashion and with $0.2 \mathrm{~mm}$ layer height since these parameter values gave the optimal mechanical properties [15-17]. Infill percentage was set to a theoretical 100\%. Printing speed and extrusion temperature were kept constant for the whole part and were defined according to the Doehlert method. A Doehlert network with two experimental designs, including ten experiments, was used to evaluate the tensile properties regarding printing speed from 15 to $105 \mathrm{~mm} / \mathrm{s}$ in seven levels and extrusion temperature from 190 to $240{ }^{\circ} \mathrm{C}$ in three levels according to Ginoux $e t$ al. work [12]. One should note that PLA7 was not printed because of its rigidity. The PLA7 filament did not accept the strain applied by the roller driving the filament towards the printing head. The broken filament was then impossible to print with FFF and only PLA0 and nanocomposites loaded under $5 \mathrm{wt} \%$ were printed.

To compare tensile properties of printed parts to fully infill parts manufactured by a formative process, materials in pellet form after compounding were injected moulded into 5A-type specimens with a Babyplast 6/10P equipped with an $18 \mathrm{~mm}$ diameter piston and a Moullo X Dry Air to preheat pellets at $70^{\circ} \mathrm{C}$ and keep them moisture-free before injection moulding at $200{ }^{\circ} \mathrm{C}$

\subsection{Characterisation tests}

Before each measurement, all samples were dried at $80{ }^{\circ} \mathrm{C}$ under vacuum $(-72 \mathrm{cmHg})$ for $3 \mathrm{~h}$.

TGA were performed for each formulation and after each process to assess the thermal stability regarding formulation and the remaining MMT mass fractions through successive extrusions. A TG209 F3 from Netzsch was used. Samples were heated from $25{ }^{\circ} \mathrm{C}$ to $800{ }^{\circ} \mathrm{C}$ at a $20 \mathrm{~K} / \mathrm{min}$ heating rate under nitrogen flow of $3 \mathrm{~mL} / \mathrm{min}$. The MMT mass fraction was controlled by thermogravimetric analysis from residue after degradation at $800{ }^{\circ} \mathrm{C}$ for 10 min under air flow for pellets, filaments, and tensile test specimens. Each measurement is repeated three times.

Calorimetric measurements were performed on filaments to assess the behaviour of nanocomposites during melting and their temperature of use. For this purpose, only the first heating is presented in this study. A DSC204 F1 from Netzsch was used and calibrated with Indium standard. Samples were analysed from 25 to $210^{\circ} \mathrm{C}$ at a $10 \mathrm{~K} / \mathrm{min}$ heating rate three times for standard deviation calculation. Equation (1) was used to calculate the crystallinity rate, $\chi_{c}$.

$$
\chi_{c}=\frac{\Delta H_{m}-\Delta H_{c c}}{\Delta H_{m}^{0}} \times \frac{1}{1-\Phi_{m}}
$$

$\Delta H_{m}$ is the measured melting enthalpy, $\Delta H_{c c}$ the measured cold crystallisation enthalpy, $\Delta H_{m}^{0}$ the melting enthalpy of a theoretical $100 \%$ crystalline PLA (93 J/g [18]), and $\Phi_{m}$ the MMT mass fraction.

PLA molar masses were determined by GPC for each formulation after compounding to determine the molar mass degradation regarding MMT mass fraction. A multidetector 390-LC apparatus from Varian with refractive index detectors was used with two $300 \times 7.5 \mathrm{~mm}$ columns of PLgel $5 \mu \mathrm{m}$ MIXED-D. Chloroform was used as a solvent for the samples, and as an eluent. Columns and detectors were held at $30{ }^{\circ} \mathrm{C}$. Sample solutions were prepared to concentrations of approximately $2 \mathrm{mg} / \mathrm{mL}$, and filtered with polytetrafluoroethylene filters with $0.45 \mu \mathrm{m}$ pores. Weight average molar masses are measured regarding polystyrene standards.

Tensile tests were conducted for each formulation, after 3D printing or after injection-moulding to compare the difference of tensile properties between additively manufactured materials and materials manufactured by a conventional formative process. 3D printed samples were tested at the ten configurations of the Doehlert network for mechanical optimisation. An Instron 33R 4204 apparatus was used with $1 \mathrm{kN}$ load at a $5 \mathrm{~mm} / \mathrm{min}$ constant tensile speed. Data were treated with Bluehill software. All measurements were repeated at least five times for mean response and standard deviations. Dixon tests $(Q-$ tests) were performed to eliminate aberrant values. Models associated to Doehlert designs were calculated after data processing and statistically checked by analysis of variance.

Capillary rheology data were obtained after compounding to assess the effect of MMT mass fraction on the rheological behaviour thanks to a Rosand apparatus with two $1 \mathrm{~mm}$ capillary dies of 0.25 and $16 \mathrm{~mm}$ in length at $190{ }^{\circ} \mathrm{C}$. Bagley and Rabinovitsch corrections were used to get viscosity as a function of the shear rate. Each formulation was measured at least two times, and mean values were obtained.

The microstructure was determined for each formulation and after each process to observe the developed MMT morphology regarding the formulation and the successive extrusions. A D8 Advance X-ray diffractometer was used at ambient temperature with a $\mathrm{Cu} \mathrm{K}_{\alpha} \mathrm{X}$-ray source of $0.15406 \mathrm{~nm}$ wavelength, generated at $40 \mathrm{kV}$ and $40 \mathrm{~mA}$. Measurements were performed with a $2 \Theta$ range between 2 and $10^{\circ}$ with 2 s steps of $0.06^{\circ}$. Samples were in a powder form using a ZM1000 
ultra-centrifugal mill under liquid nitrogen. TEM tests were conducted by the Institute of Materials Science of Mulhouse (IS2M, France) with a Philips CM200 at an acceleration voltage of $200 \mathrm{kV}$. TEM samples $90 \mathrm{~nm}$ thick were prepared using a Leica EM UC7 ultramicrotome at room temperature.

\section{Results and discussion}

\subsection{Nanocomposite behaviour in processes}

- PLA molar mass stability

Weight average molar mass of PLA in nanocomposite pellets after compounding is depicted in function of the MMT mass fraction in Figure 2. Considering experimental errors, a linear decrease with the mass fraction is observed, which was attributed to the presence of interlayer water. As the OMLS was not dried to increase the basal spacing and to improve the intercalation/exfoliation process [19], interlayer water could be released in the matrix during compounding and cause PLA to degrade if water content has reached $250 \mathrm{ppm}$ [20]. The decrease of the weight average molar mass could be essentially assigned to the degradation of PLA during compounding of the masterbatch since it was dried before dilution and compounding of nanocomposites from PLA05 to PLA7. This dilution could follow a proportionality rule and would explain the linear dependency. The decrease of PLA molar mass could significantly modify the rheological and thermomechanical properties of materials and should be reminded as an indirect effect of MMT on nanocomposite properties. Indeed, among others, direct effects of MMT are an increase of Young's modulus [21], a higher thermal stability to degradation [22], and a thickening effect on polymer rheology [23], while a decrease of molar mass implies lower tensile properties, a lower stability to degradation [24] and a reduced viscosity [25].

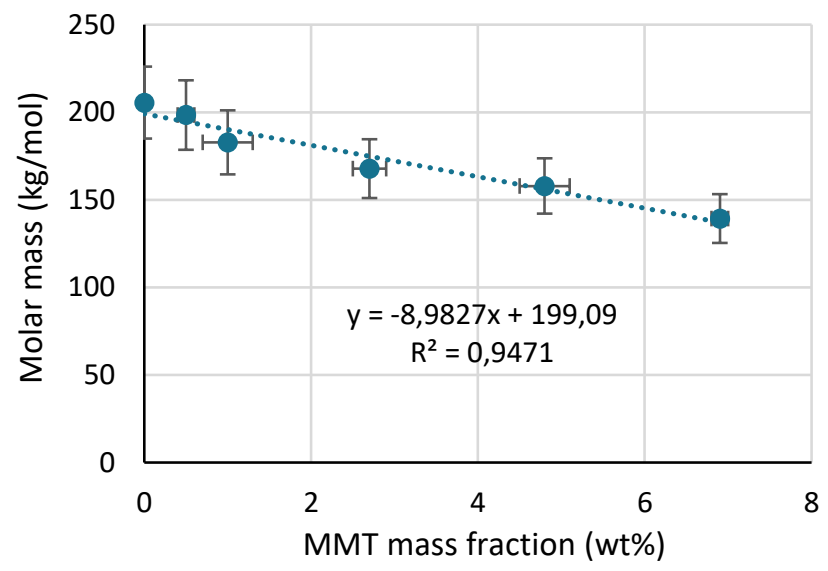

Figure 2. Linear decay of the weight average molar mass of PLA, as measured by GPC, with the addition of OMLS during the elaboration of nanocomposites. Horizontal bars depict the standard deviations of MMT mass fractions as determined by TGA. Vertical bars are an assumed error of $10 \%$ on molar mass measurements.

- Evolution of MMT morphology

Table 1 summarises XRD data and the intercalation state of MMT platelets in nanocomposites after each process step, namely compounding, filament shaping and printing by FFF (compound data from a previous work [11]). PLA5 presents a mixed morphology with the presence of peaks attributed to tactoids and intercalated platelets. Compared to the basal spacing of C30B, $1.87 \mathrm{~nm}$, tactoids of PLA5 show a lower average basal spacing of $1.68 \mathrm{~nm}$. This reduced basal spacing could be due to either an organo-modifier departure towards the PLA matrix or a beginning of its degradation leading to shorter hydrocarbonated chains and narrower interlayer spacing. By diluting the MMT loading, PLA3 only shows a peak of intercalated platelets, while XRD peaks for PLA05 and PLA1 were both not observable. The absence of peaks means either a fully exfoliated state or a low particle density to emit a signal in XRD analysis [25]. To verify the exfoliation state of PLA05 and PLA1, direct imaging is needed. Figure 3 illustrates the MMT morphology of PLA05 and PLA1 from TEM micrographs. It appears the two diluted nanocomposites, namely PLA05 and PLA1, consist of exfoliated platelets, except for rare intercalated platelets in PLA1 after compounding, totally absent after filament shaping and printing. Both formulations can be qualified as having reached a good exfoliation level. Therefore, the intercalation/exfoliation level increases as the MMT loading decreases. The observation is consistent with the work of Aubry et al. [26] reporting that 
particles tend to stay in an aggregate form after a maximum packing volume fraction, around 1 vol\%, which returns an intercalation process less efficient with semi-diluted nanocomposites.

Table 1. Comparisons of Bragg angle, intensity, and basal spacing of MMT platelets in C30B and nanocomposites at different MMT contents from 0.5 to $5 \mathrm{wt} \%$ and between each process step, as determined by XRD measurements.

\begin{tabular}{cccccc}
\hline \multirow{6}{*}{ Control } & $\begin{array}{c}\text { Sample } \\
\text { name }\end{array}$ & $\begin{array}{c}\mathbf{2 \Theta} \\
\left({ }^{\circ}\right)\end{array}$ & $\begin{array}{c}\text { Intensity } \\
(\mathbf{c p s})\end{array}$ & $\begin{array}{c}\boldsymbol{d}_{\mathbf{0 0 1}} \\
(\mathbf{n m})\end{array}$ & Comments \\
\hline \multirow{5}{*}{ Compound } & 4.73 & 18207 & 1.87 & Tactoid \\
\hline \multirow{5}{*}{ Filamenten } & PLA05 & $/$ & $/$ & $/$ & $/$ \\
& PLA1 & $/$ & $/$ & $/$ & $/$ \\
& PLA3 & 2.89 & 323 & 3.05 & Intercalated \\
& PLA5 & 2.59 & 153 & 3.41 & Intercalated \\
& PLA5 & 5.26 & 698 & 1.68 & Tactoid \\
\hline \multirow{5}{*}{ Print } & PLA05 & $/$ & $/$ & $/$ & $/$ \\
& PLA1 & $/$ & $/$ & $/$ & $/$ \\
& PLA3 & $/$ & $/$ & $/$ & $/$ \\
& PLA5 & 5.03 & 499 & 1.76 & Tactoid \\
& PLA5 & 7.52 & 170 & 1.17 & Tactoid \\
\hline & PLA05 & $/$ & $/$ & $/$ & $/$ \\
& PLA1 & $/$ & $/$ & $/$ & $/$ \\
& PLA3 & $/$ & $/$ & $/$ & $/$ \\
& PLA5 & 2.59 & 81 & 3.41 & Intercalated \\
& PLA5 & 5.20 & 300 & 1.70 & Tactoid \\
& PLA5 & 7.69 & 74 & 1.15 & Tactoid \\
\hline
\end{tabular}

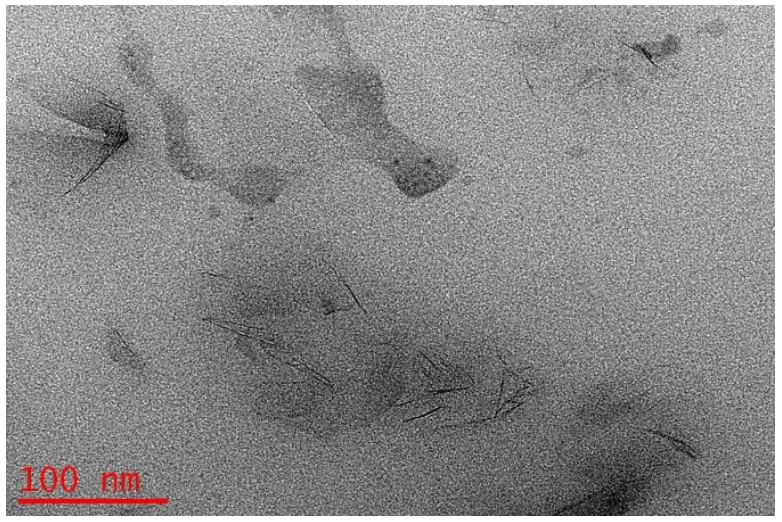

(a)

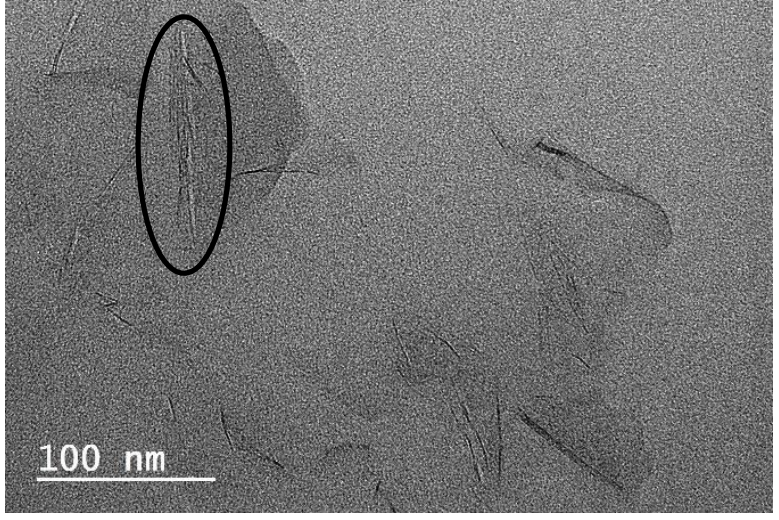

(b)

Figure 3. TEM micrographs of (a) PLA05 and (b) PLA1 nanocomposites after compounding. Black oval points out some observed intercalated platelets in PLA1.

Figure 4 shows the evolution of MMT morphology after each process step for PLA3 and PLA5. PLA3 essentially consists of intercalated platelets after compounding and exfoliated ones after filament shaping and printing. The evolution towards a more exfoliated state is consistent with the disappearance of peaks in XRD for PLA3 after filament shaping and printing (see Table 1). Similarly, PLA5 TEM micrographs after compounding (Figure 4b) indicates the presence of tactoids and some intercalated platelets and the presence of the three morphologies after filament shaping and printing (Figures $4 \mathrm{~d}$ and 4f), namely tactoids and intercalated and exfoliated platelets. These observations are consistent with the decrease of intensity of XRD peaks from tactoids in PLA5 after each process step and the appearance of a peak from intercalated platelets after printing (Table 1). Therefore, the successive extrusions tend to increase the intercalation/exfoliation state, which concurs with the works of Vaia and Giannelis [27] since the intercalation is a kinetic process. The increase of time spent in a molten state would then lead the nanocomposites to evolve towards a more intercalated or exfoliated state. However, a second XRD peak is observed for PLA5 around $7.6^{\circ}$ after filament shaping and printing. The corresponding basal spacing is around 1.15-1.17 nm and close to that of native MMT $(1.17 \mathrm{~nm})$ [28]. Consequently, PLA5 is shared between (1) an evolution towards a more intercalated/exfoliated morphology with the successive extrusions and lengthening of time spent in a molten state, and (2) the organo-modifier departure from the interlayer spacing or its 
degradation leading to more aggregated particles. The first phenomenon would develop the thermomechanical properties of the materials, while the second one would limit them [14]. More precisely, the trend to aggregate could be an issue during printing by clogging the nozzle at high MMT loadings. Nevertheless, the reduced interlayer spacing is not accompanied by an increase of the number of particles in a stack, and the phenomenon seems then limited on TEM micrographs (Figure 4f) compared to the evolution towards a more intercalated state.

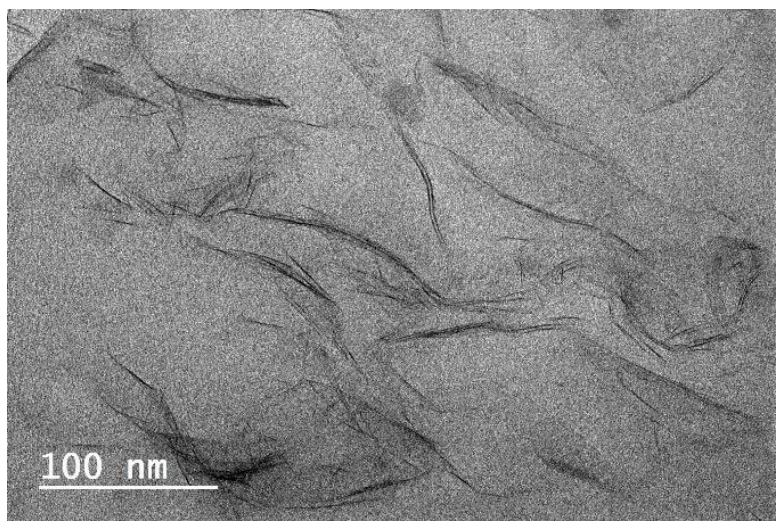

(a)

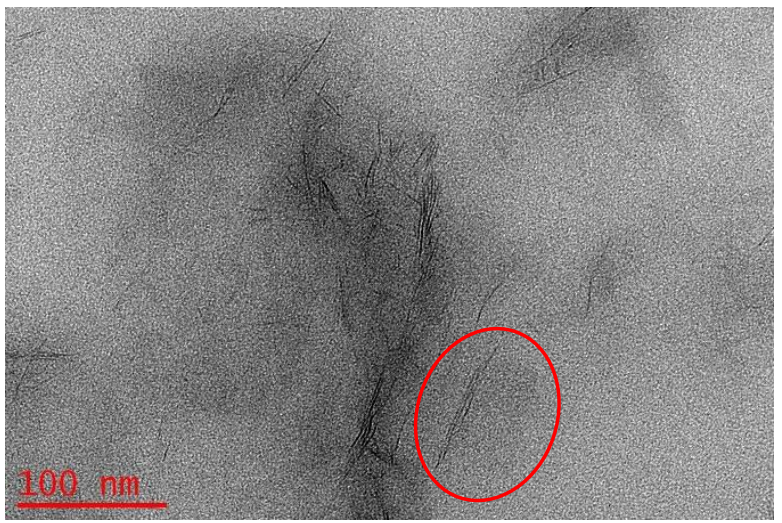

(c)

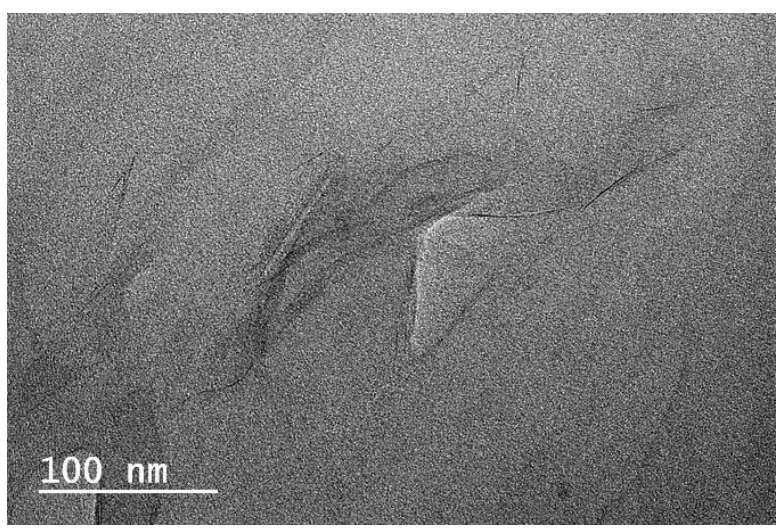

(e)

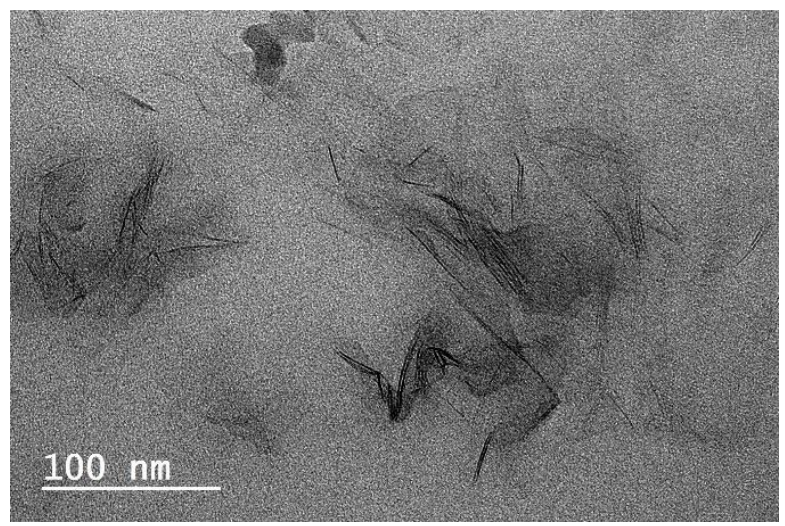

(b)

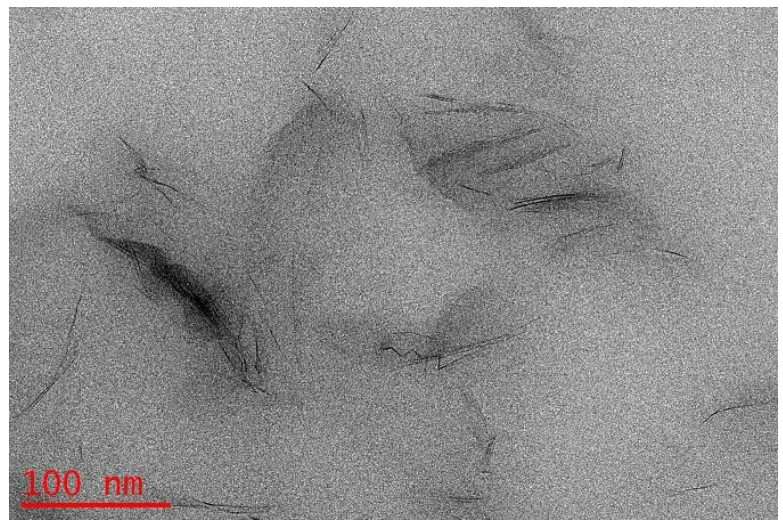

(d)

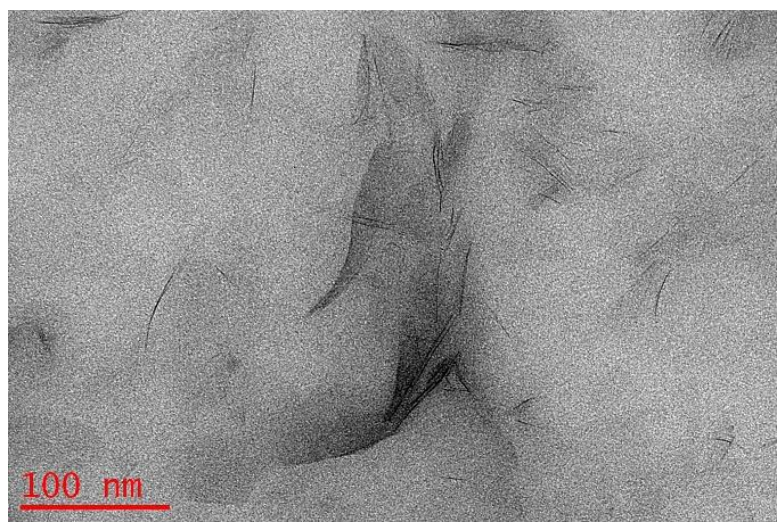

(f)

Figure 4. TEM micrographs of (a,c,e) PLA3 and (b,d,f) PLA5 nanocomposites after (a,b) compounding, (c,d) filament shaping, and (e,f) printing.

- Evolution of MMT loading

Figure 5 illustrates the evolution of MMT loading in nanocomposites after each process step. The MMT mass fractions appear close to target values after compounding: $0.5 \pm 0.1,1.0 \pm 0.3,2.7 \pm 0.2$, and $4.8 \pm 0.3 \mathrm{wt} \%$ for, respectively, PLA05, PLA1, PLA3, and PLA5. A mild lower value for PLA3 and PLA5 could be assigned to an MMT loss during compounding. A more visible decrease of around 10\% is observable for PLA3 and PLA5 from beginning to end, mostly assigned to the 
filament shaping process; MMT mass fractions were $2.5 \pm 0.1 \mathrm{wt} \%$ for PLA3 and $4.3 \pm 0.1 \mathrm{wt} \%$ for PLA5. Regarding standard deviations, FFF process does not significantly decrease the MMT mass fractions, with measured values being 2.4 $\pm 0.2 \mathrm{wt} \%$ for PLA3 and $4.2 \pm 0.1 \mathrm{wt} \%$ for PLA5. Therefore, one should overload the polymer matrix by around $10 \%$, depending on the used shaping process, to compensate the loss of MMT during filament shaping, and eventually during compounding. As a second consequence of these observations, nanocomposites do not present a high clogging risk for long duration prints since FFF process does not seem to remove MMT from materials to machine. Then, viability of nanocomposites with FFF is not expected to be limited by an accumulation of MMT in the printing head.

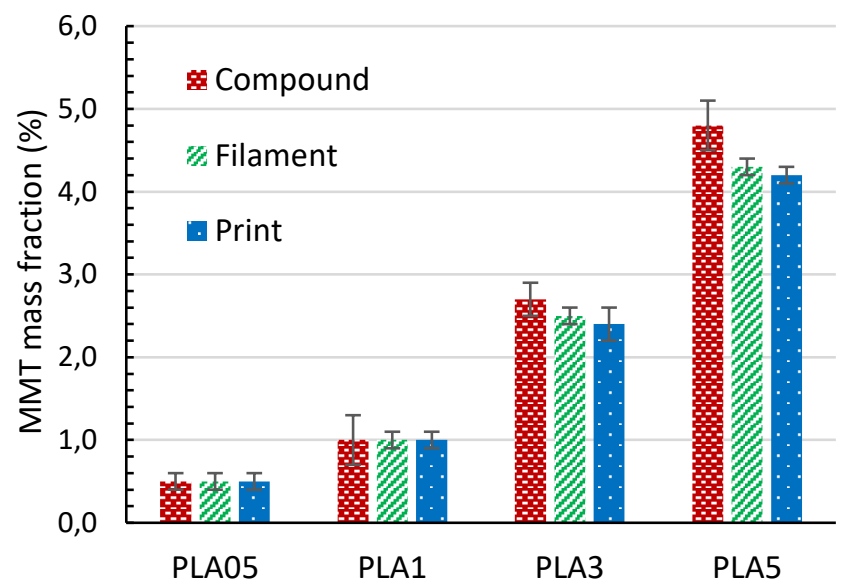

Figure 5. Evolution of MMT mass fraction after compounding, filament shaping and printing for each formulation. Vertical bars represent the standard deviations calculated from MMT mass fraction measurements as determined by TGA.

- Nanocomposite behaviour during melting inside printing head

Figure 6 shows the DSC analysis that informs on the behaviour of nanocomposites during melting, which would determine the temperature of use. As the filaments after shaping were quenched, the polymer matrix was amorphous. The temperature of use of nanocomposites in FFF could be then just above the softening temperature, above the glass transition after $76^{\circ} \mathrm{C}$ (see Figure 6). However, DSC measurements also indicate that nanocomposites go through a cold crystallisation, starting around $89^{\circ} \mathrm{C}$, which would increase the viscosity of materials and cause a greater probability of nozzle clogging. Cold crystallisation temperature of nanocomposites is lower than that of neat PLA and could be caused by a nucleating effect of the MMT [29], which would ease the cold crystallisation mechanism. A decrease of the molar mass with the clay loading (see Figure 2) could also be an explanation to the ease of nanocomposite to crystallise because of the greater mobility of polymer chains [30]. Around $160{ }^{\circ} \mathrm{C}$, an exothermic peak is visible, which is attributed to a disorder-to-order transition $[24,31]$, and is followed by the melting of PLA around $180{ }^{\circ} \mathrm{C}$. The variation in melting temperature between the different formulations is not significant, which is consistent with other PLA-based nanocomposites, namely PLA/graphene for instance [32]. However, greater melting temperatures were also reported with other nanocomposites, namely polyether block amide/graphene nanocomposite, while adding nanofiller [33]. From those two studies, such difference is explained by the nanofiller/matrix interaction and the degree of intercalation/exfoliation of the nanoplatelets [32,33]. In the case of PLA-based nanocomposites, when observing the morphology of PLA/OMLS (see Table 1 and Figure 3), the low number of platelets in diluted systems and the transition to an intercalated morphology in the semi-diluted systems are not sufficient to impact the melting process (see Figure 6). Consequently, the printing temperature should be above the melting temperature, which ends up after $190{ }^{\circ} \mathrm{C}$ independently of the formulation, to avoid a potential clogging risk because of the crystallisation. The temperature of use of all PLA/OMLS nanocomposite filaments is recommended to be above 190 ${ }^{\circ} \mathrm{C}$. 


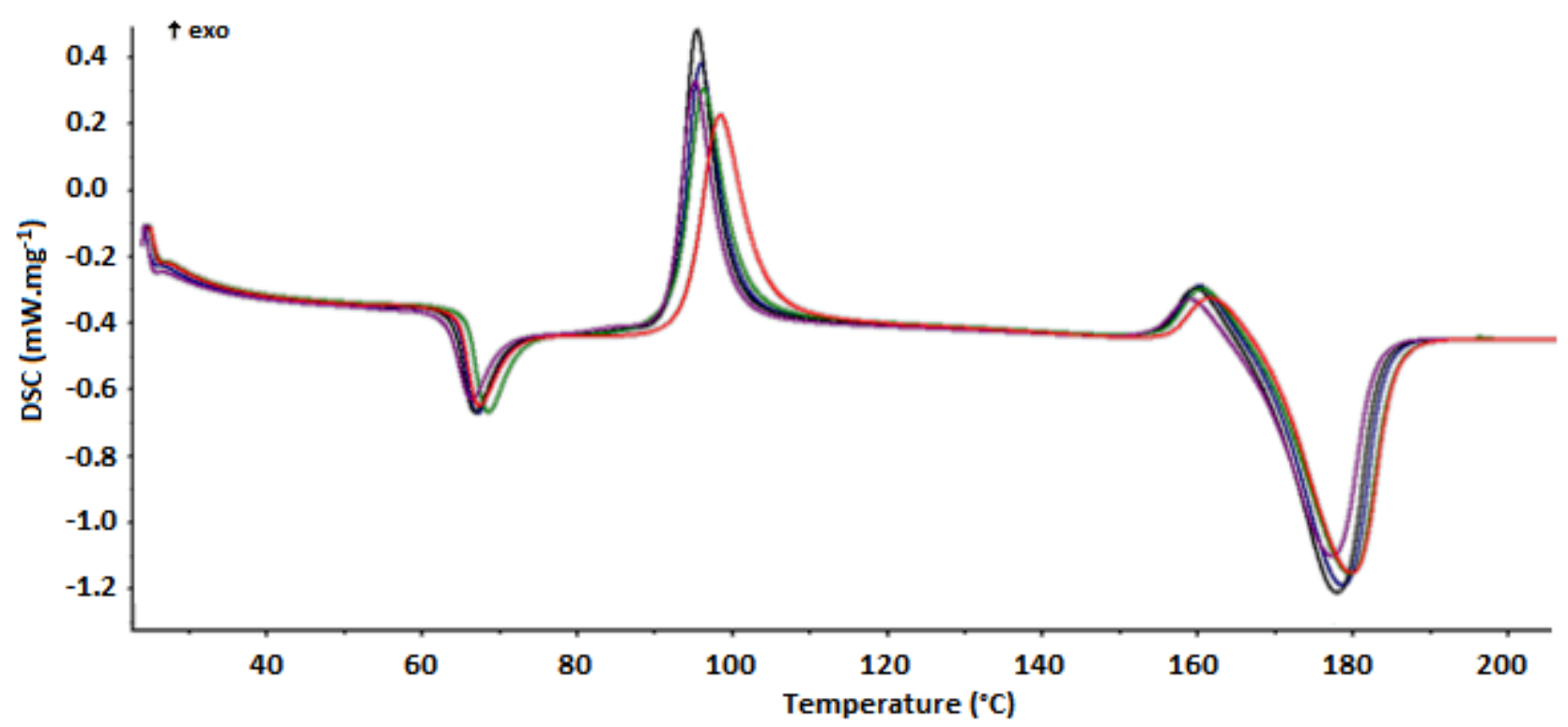

Figure 6. DSC thermograms of PLA0 (red), PLA05 (green), PLA1 (blue), PLA3 (black), and PLA5 (purple) after filament shaping at the first heating.

- Printability

Printability is conditioned by the possibility of use of filaments and the rheological behaviour of nanocomposites in extrusion process. Firstly, as written in section 2. Materials and methods, PLA7 cannot be printed because of the filament stiffness increasing with the MMT loading, as already reported by Lee et al. [34]. Secondly, the increase of MMT mass fraction is expected to increase the viscosity [10], which would cause the impossibility of the materials to be printed [8]. Nevertheless, semi-diluted nanocomposites were observed with a lower viscosity compared to that of neat polymer in nonlinear rheology [35,36]. These authors suggested a possible slip between the matrix and exfoliated MMT as well as a decrease in molar mass with the addition of fillers during mixing. Despite the increase of viscosity with the addition of fillers, it appeared according to Ginoux et al. study [11] that the decrease in viscosity is mainly attributed to greater activation energies with semi-diluted nanocomposites and to the PLA molar mass degradation as observed in Figure 2. Therefore, the viscosity in function of the MMT loading increases after the first addition of MMT, then decreases up to be lower than that of neat PLA after $3 \mathrm{wt} \%$ of MMT (see Figure 7). The decrease is more pronounced with higher printing temperature since the dependency of viscosity on temperature is greater with semi-diluted nanocomposite $(>1 \mathrm{wt} \%)$ [11]. This observation is in agreement with the printing failure rate noted during experimental design. On the entire Doehlert network, consisting of ten experiments, PLA0 showed a global printing failure rate around 10\%, while that of PLA05 and PLA1 was between 20 to $40 \%$ in function of the configuration. The higher failure rate is consistent with a higher probability of nozzle clogging because of the thickening effect by the filler with diluted systems. On the contrary, PLA3 and PLA5 showed a drastic decrease of printing failure rate, below $10 \%$, which is, again, consistent with a thinning effect by the filler with semi-diluted systems. The printability is then improved for nanocomposites loaded at more than $3 \mathrm{wt} \%$ of MMT due to a direct and indirect thinning effect by the filler on the molten materials but worsened at more than $7 \mathrm{wt} \%$ due to the stiffness of the filaments. 


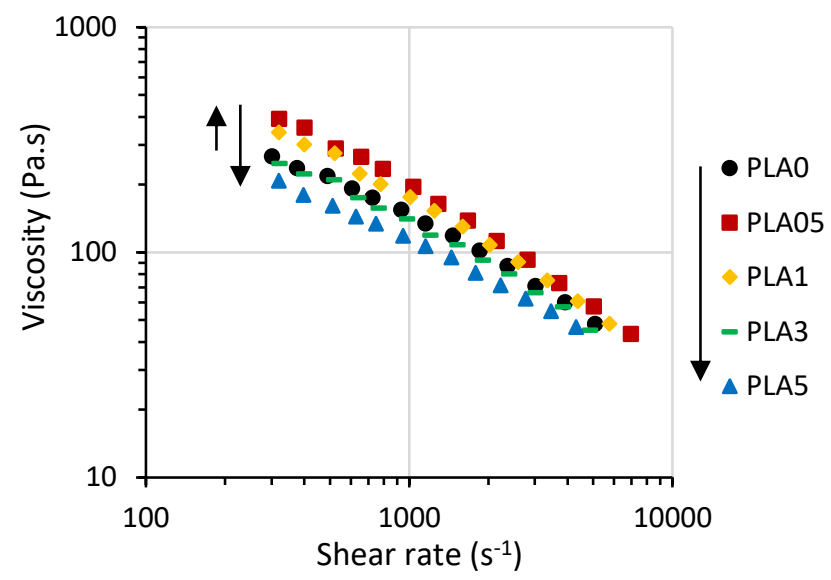

Figure 7. Rheogram plotting the viscosity against the shear rate for PLA0 to PLA5 at $190{ }^{\circ} \mathrm{C}$. Black arrows show the increase followed by the decrease of viscosity with the increase of MMT loading.

\subsection{Thermomechanical optimisation}

- $\quad$ Thermal stability

Figure 8 depicts the evolution of thermal degradation temperatures of PLA with MMT loading. The more the MMT is added to PLA, the lower the onset of degradation, and the greater the remaining residue. Actually, the addition of plateletlike fillers was reported in the literature to shift the onset of degradation towards higher temperatures by inducing fewer effective collisions to trigger the intra- and inter-molecular degradation phenomena [21,22]. However, Saiter et al. [24] also noticed a decrease of the onset of degradation with the addition of MMT, which was supposed to be due to the predegradation of the polymer matrix by the addition of filler during compounding. As seen in Figure 2, the addition of OMLS to the PLA reduced the matrix molar mass and is consistent with the assumptions from Saiter et al. study [24].

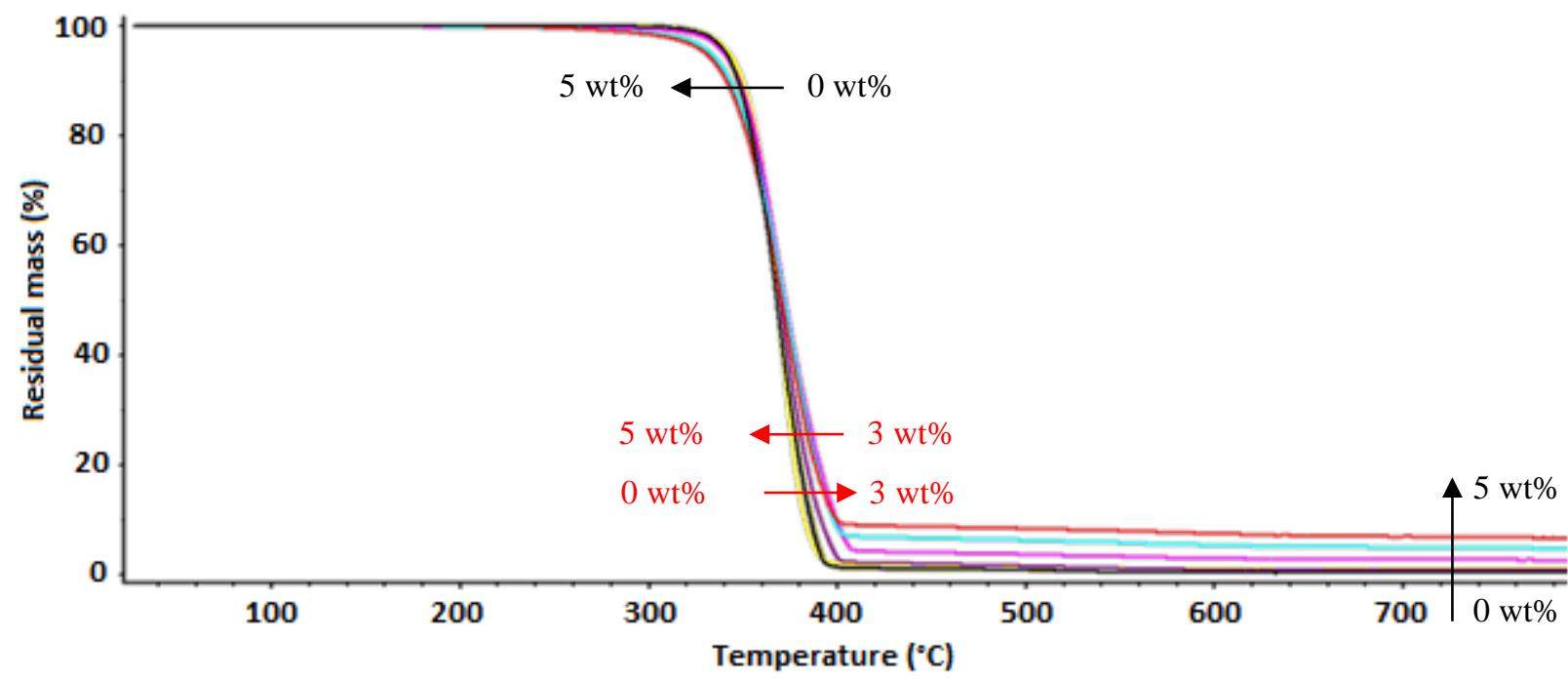

Figure 8. TGA thermograms at a $10 \mathrm{~K} / \mathrm{min}$ heating rate from PLA0 to PLA5. Black arrows show the variation of thermal degradation onset and of residual mass after degradation with the increase of MMT loading in PLA.

The endset of degradation firstly indicates an increase with the addition of MMT from 0 to $3 \mathrm{wt} \%$, then a decrease afterwards. The degradation temperature range appears more reliable for discussion as the endset is dependent on both the onset and the kinetics of thermal degradation. In that way, the degradation temperature range is around $38.6 \pm 1.4{ }^{\circ} \mathrm{C}$ for diluted systems (from PLA0 to PLA1 included) and reaches $49.2 \pm 0.5{ }^{\circ} \mathrm{C}$ for semi-diluted systems (PLA3 and PLA5). Krishnamachari et al. [37] had suggested the migration of MMT to explain the increase of thermal stability since the filler would create a protective physical barrier. A direct visual observation after TGA of diluted systems showed the presence of a white powder with granulometry in the micrometer range, while semi-diluted ones had a different aspect (see Figure 
9). PLA3 and PLA5, after TGA, showed a remaining porous and friable structure of the size of the pellets before thermal degradation. This structure could act as a physical barrier. As observed in Figure 9b, blisters are visible on the external surface and could reflect the overpressure by the accumulation of degraded materials. This phenomenon would result in a slower kinetics of thermal degradation by slowing down the departure of degraded materials to the outside of the system. The formation of such structure could be attributed to the relatively high amount of MMT in semi-diluted systems, which had previously indicated an increase of the filler-filler interactions at the expense of filler-matrix ones with a less exfoliated morphology (see section 3.1.), leading to a percolation. Consequently, the thermal stability is indirectly reduced by the predegradation of the PLA matrix by the OMLS inherent to the compounding process but is improved by a barrier-structure during degradation after a percolation threshold. After this critical mass fraction, kinetics do not seem modified and an optimal MMT loading is expected around $3 \mathrm{wt} \%$ to increase the thermal stability by the barrier-structure formation without reducing it excessively by the pre-degradation.

(a)

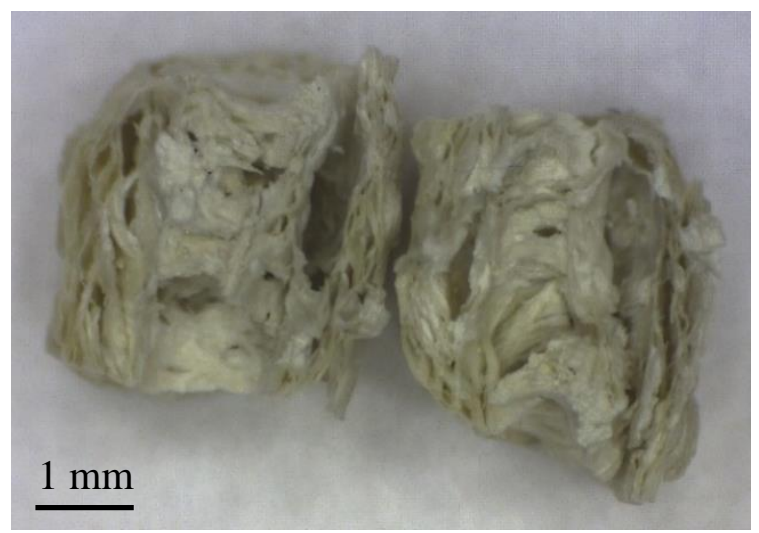

(b)

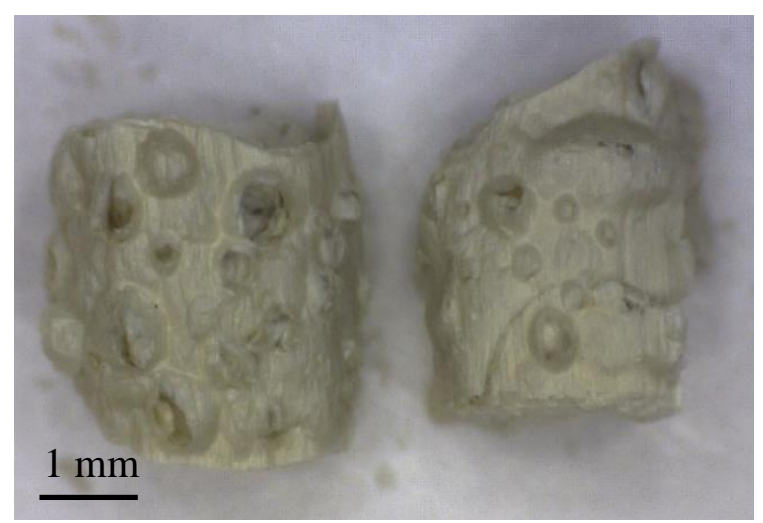

Figure 9. Photographies of a PLA5 pellet after thermal degradation, cut in half with (a) the inside view and (b) the outside view.

- Optimisation of tensile properties by Doehlert designs

Elongations at break and at ultimate tensile strength were treated by Doehlert method, but experimental standard deviations were high, leading to low significance on all the coefficients and models. Therefore, printing speed and extrusion temperature have been concluded with no impacting effect on elongations. However, by taking the mean value of elongation at break from all the Doehlert design configurations for a same nanocomposite, then by comparing them in function of the MMT loading, the effect of the formulation is observable. The variation of elongation at break in function of the MMT loading for printed samples is compared that of injected moulded samples in Figure 10. One could see that, independently of the process, diluted systems show greater elongations at break than that of neat PLA, while semi-diluted ones show the lowest elongations. The observation is consistent with literature: Lai et al. [38] reported an extended elongation at break for PLA charged with $1 \mathrm{wt} \%$ of MMT compared to more loaded nanocomposites. This phenomenon is assigned to a plasticised interphase surrounding nanoparticles. This interphase modifies the micromechanical properties when abundant, which would be the case when particles are smaller than $100 \mathrm{~nm}$ [39]. With the use of MMT as a filler, 
only exfoliated platelets correspond to particles smaller than $100 \mathrm{~nm}$, and intercalated or aggregated ones would not develop abundant plasticised interphase. As observed previously, only PLA05 and PLA1 have indicated a fully exfoliated state and would explain the extended elongations at break.

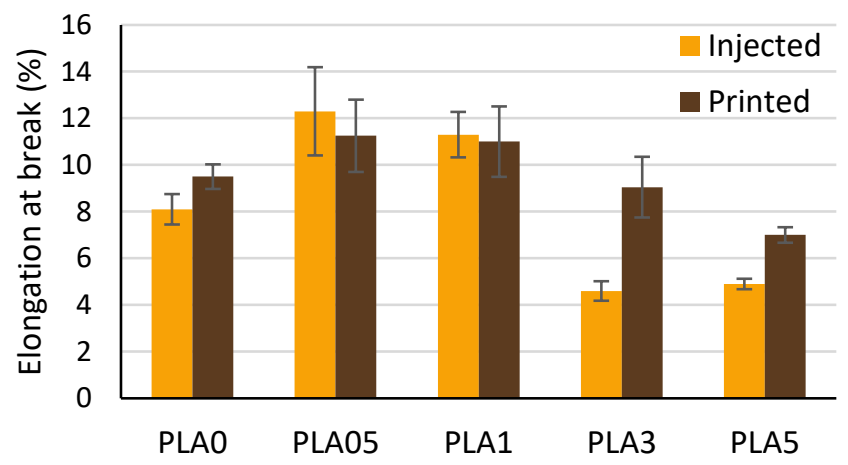

Figure 10. Comparison of the elongations at break of 5A-type tensile test specimens manufactured by injection moulding (in light orange) and by FFF (in dark brown) in function of the MMT loading. Vertical bars represent the standard deviations on ten measures for injected moulded specimens and on fifty measures for printed specimens (five measures at each of the ten configurations of the Doehlert network).

For semi-diluted systems (PLA3 and PLA5), MMT stiffens the PLA-based materials and the elongation at break is reduced. However, the decrease is less pronounced for printed specimens than for injected ones that have half the elongations of neat PLA (Figure 10). The MMT loss during filament printing and the more exfoliated/intercalated morphology with the successive extrusions could be an explanation but did not seem to evolve to the point of increasing the elongation at break of the printed tensile test specimens from $+42 \%$ (PLA5) to $+97 \%$ (PLA3) compared to the injected samples (see section 3.1.). A more relevant explanation may come to the mesostructure inherent to the FFF process. The interlayer adhesion is known to be weak in additively manufactured parts, which brings brittleness to the parts [40]. This phenomenon is even more pronounced with the addition of filler as the nanocomposites have greater viscosities when shear rate is null, right after deposition, due to a percolation threshold [11], which causes a quite weak adhesion of the deposited filaments [41]. The enhanced elongation at break is then counter-intuitive, but an explanation stands in the de-adhesion of the rasters in a same layer, rather than between layers [40]. As the materials are deposited in a $45^{\circ} /-45^{\circ}$ fashion and due to the structure consisting of deposited filaments, the rasters separate from each other by shearing, which realigns each raster in the load direction during tensile testing and the tensile test specimens would be able to fracture filament by filament (see Figure 11). The fracture toughness is then improved for printed samples, while injected ones have a sharper fracture for the semidiluted systems, namely PLA3 and PLA5. Therefore, the mesostructure of the printed samples would compensate the loss of elongation at break caused by the addition of a high MMT amount in PLA with the injected moulded specimens.

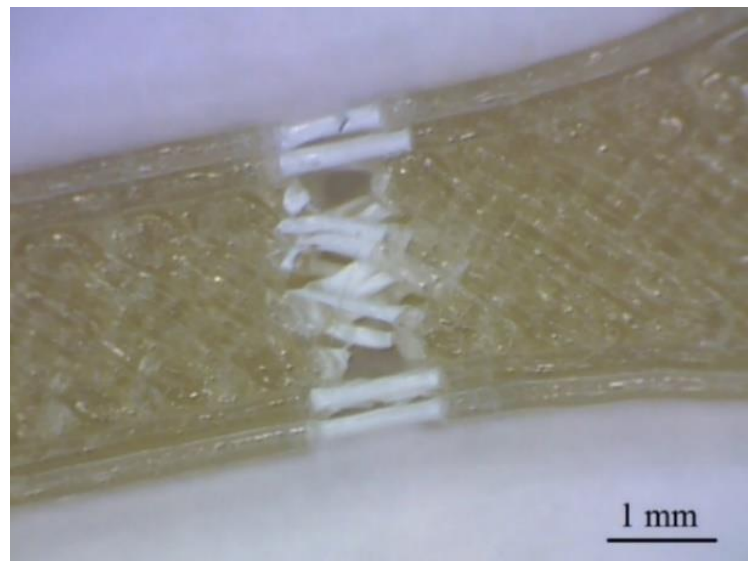

Figure 11. Photography of the fracture of a printed 5A-type tensile test specimen of PLA3 after tensile test at a $5 \mathrm{~mm} / \mathrm{min}$ tensile speed. 
Young's modulus and ultimate tensile strength were chosen as responses for models with Doehlert designs. Models globally showed a minimum or a tendency to a minimum on the first design (15 to $75 \mathrm{~mm} / \mathrm{s}$ and 190 to $\left.240{ }^{\circ} \mathrm{C}\right)$ and a maximum or a tendency to a maximum on the second design ( 45 to $105 \mathrm{~mm} / \mathrm{s}$ and 190 to $240{ }^{\circ} \mathrm{C}$ ) as already reported in Ginoux et al. work [12] because of the numerous phenomena modifying tensile properties. To compensate the loss of mechanical properties from formative to additive process, maximum responses are desired for optimisation and can be calculated from Doehlert quadratic mathematical model. Predicted maximum responses and factor values corresponding to the maximum response are summarised in Table 2. Printing speed and extrusion temperature tend to increase then to decrease with the addition of OMLS in PLA (see Table 2). The variation is analogous to that of viscosity (see Figure 7). Because of several deposition defects depending on flowability and instabilities with the printing speed, an optimal viscosity is expected [12], and is an explanation to the increase then to the decrease of printing speed, correlated to the shear rate. As nanocomposites show shear-thinning behaviour (see Figure 7), higher shear rates compensate the higher viscosities with diluted systems (PLA05 and PLA1) compared to semi-diluted systems (PLA3 and PLA5) or neat PLA. One should note that extrusion temperature tends to increase with printing speed. An explanation stands in the decrease of residence time in the heating nozzle with higher flow rates, which is not favourable to an optimal heat transfer. Although higher shear rates could self-heat the molten materials in opposition, this phenomenon does not compensate the lack of heat transfer and would need a compensation by a higher extrusion temperature setpoint.

Table 2. Maximum responses predicted by Doehlert design with the Young's modulus $(E)$ and the ultimate tensile strength $\left(\sigma_{M}\right)$ as response in function of the MMT loading and with the printing speed and extrusion temperature associated to the predicted response. Prediction is not presented if the maximum is out of the Doehlert network.

\begin{tabular}{ccccc}
\hline Sample & Property & $\begin{array}{c}\text { Speed } \\
(\mathrm{mm} / \mathrm{s})\end{array}$ & $\begin{array}{c}\text { Temp. } \\
\left({ }^{\circ} \mathrm{C}\right)\end{array}$ & $\begin{array}{c}\text { Prediction } \\
(\mathrm{MPa})\end{array}$ \\
\hline \multirow{2}{*}{ PLA0 } & $E$ & 67 & 212 & 1274 \\
& $\sigma_{M}$ & 73 & 209 & 64,0 \\
PLA05 & $E$ & $\sim 60$ & $>240$ & $/$ \\
& $\sigma_{M}$ & 60 & 221 & 62,2 \\
PLA1 & $E$ & 95 & 239 & 1268 \\
& $\sigma_{M}$ & $\sim 105$ & $>240$ & $/$ \\
PLA3 & $E$ & 81 & 217 & 1401 \\
& $\sigma_{M}$ & 67 & 206 & 59,6 \\
PLA5 & $E$ & 72 & 192 & 1527 \\
& $\sigma_{M}$ & $\sim 50$ & $<190$ & $/$ \\
\hline
\end{tabular}

Figure 12 compares the Young's modulus and the ultimate tensile strength between full and printed specimens at different MMT loadings. As expected, according to literature [1], tensile properties are lower for printed samples than for injected ones. The properties of the printed specimens are represented with the maximum response and the minimum response obtained throughout the Doehlert network. These responses are experimental and not predicted. These values may then extend beyond (see Table 2). The average mechanical properties over the entire network are also displayed in Figure 12 and serve as a basis for evaluating the variation of the properties with the MMT loading, independently of a specific configuration. Whatever the manufacture process, the Young's modulus is linearly correlated with the MMT loading (Figure 12a). However, the improvement of the Young's modulus with the MMT loading is enhanced for the injected specimens with a sharper slope compared to that of the printed specimens. Conversely, the maximum stress decreases linearly with the MMT loading for the printed specimens (Figure 12b), and the use of nanocomposite cannot compensate the loss of properties from injected samples to printed ones. Similarly to elongation at break, diluted systems show a synergetic effect with the development of matrix/filler interaction over filler/filler or matrix/matrix interactions, which lead to higher ultimate tensile strength compared to ultimate tensile strengths for neat PLA or semi-diluted systems. This synergetic effect is not observable with printed samples and could be hidden by the dominance of structural defects inherent to the FFF process. 
(a)

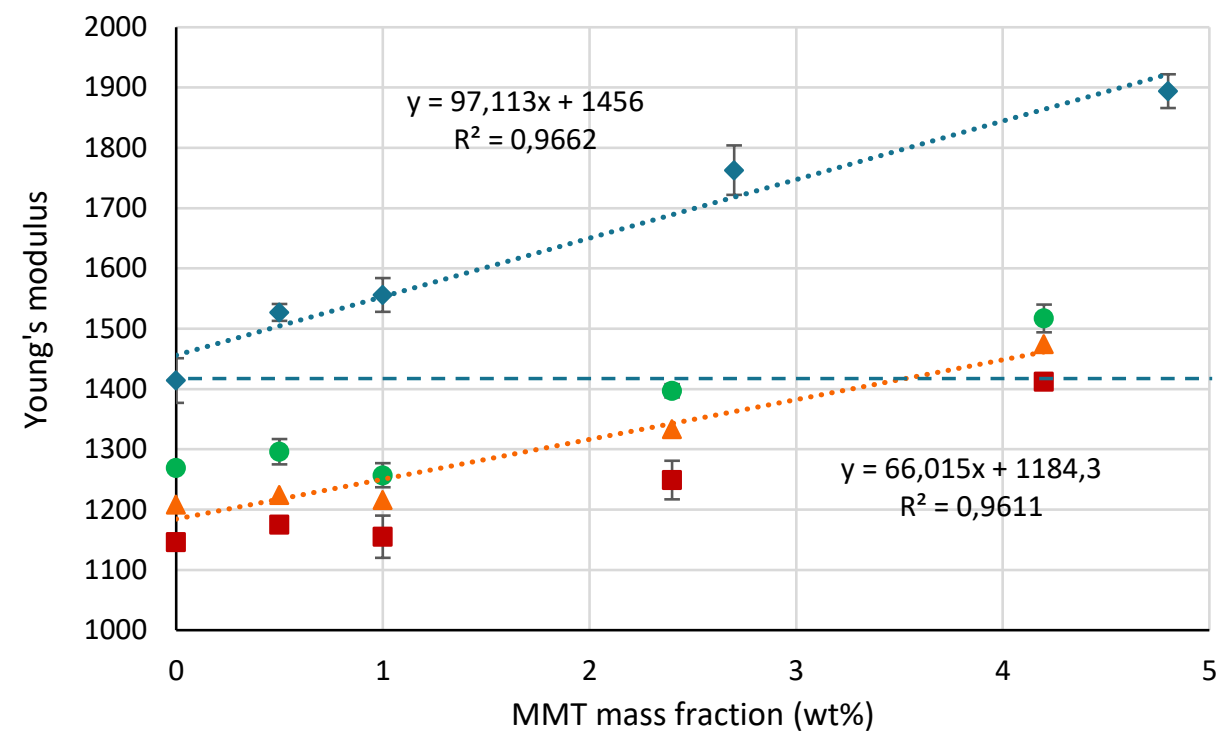

(b)

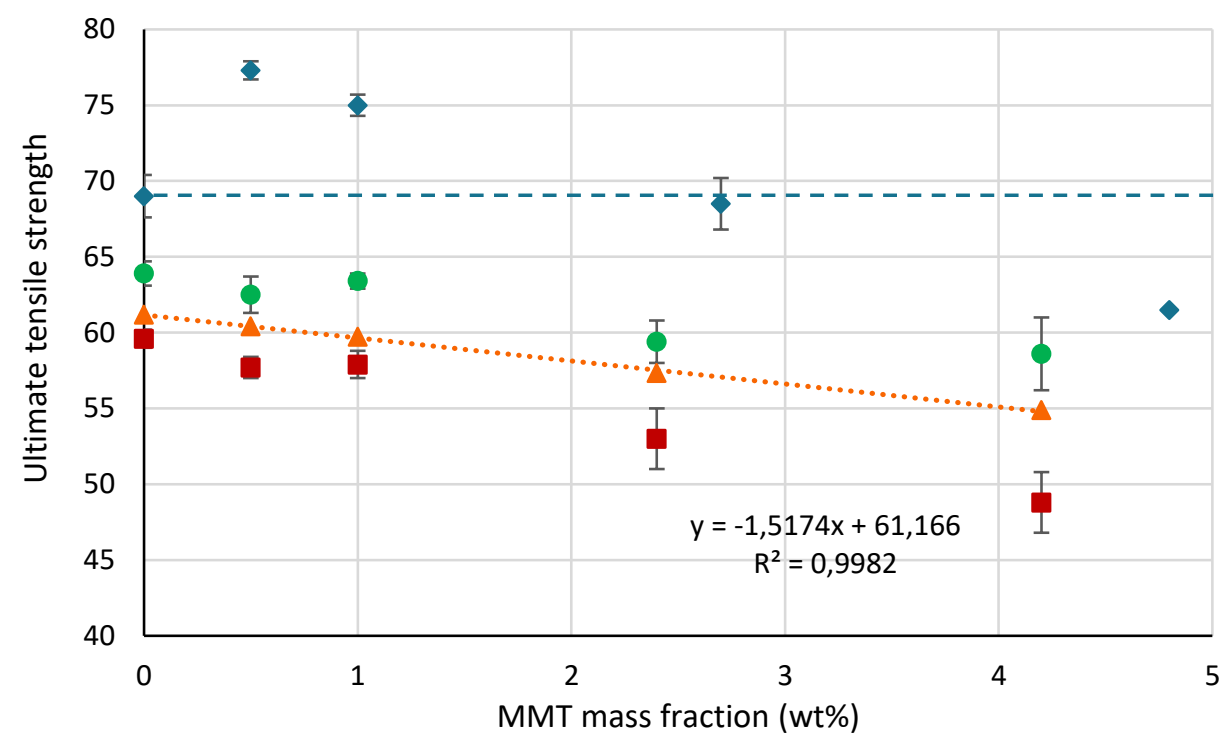

Figure 12. Comparison of (a) the Young's moduli and (b) the ultimate tensile strengths in function of the MMT mass fraction in PLA, as measured by tensile test on injected moulded 5A-type specimens (blue diamond) and on printed 5Atype specimens at the maximum response (green circle), at the minimum response (red square) and at the mean response (orange triangle) of the ten configurations from the Doehlert network. Vertical bars represent the standard deviations on ten measures for injected moulded specimens and on five measures for printed specimens.

For neat PLA, the average loss of mechanical properties between formative and additive processes is estimated around $14.6 \%$ for the Young's modulus and $11.3 \%$ for the ultimate tensile strength. However, this loss can reach respectively $19.0 \%$ and $13.6 \%$, but can also be minimised to $10.3 \%$ and $7.4 \%$ regarding the configuration in the studied Doehlert network. With the prediction from the theoretical mathematical models (see Table 2), this loss of properties can be lowered to $9.9 \%$ and $7.2 \%$ for Young's modulus and ultimate tensile strength. Consequently, the optimisation of the printing speed and extrusion temperature cannot reach alone the mechanical properties of the injected PLA0 specimens. Nevertheless, the use of the Doehlert method divides by two the loss of mechanical properties by comparing an unfavourable configuration with a favourable one. Full property compensation must then go through the use of nanocomposite. Thus, the Young's modulus of injected moulded PLA0 is approximately equal to that of PLA3 at its maximum response and that of PLA5 at its minimum response. On the other hand, because of the negative tendency of the ultimate tensile strength with the MMT loading, the minimum loss of property is achieved with the PLA0. To obtain similar properties to the PLA0 specimens manufactured by injection moulding, one should opt for the more diluted nanocomposite in MMT to avoid the loss on the ultimate tensile strength while reaching the same Young's modulus at a configuration maximising the response. Therefore, the PLA3 printed to the maximised configuration seems to be the best compromise if the optimisation of the Young's 
modulus and/or thermal stability (see Thermal stability section) has priority over the ultimate tensile strength. In addition, its elongation at break is close to that of injected PLA0. For a priority optimisation on the ultimate tensile strength, the PLA0 printed to the maximised configuration is recommended.

\section{Conclusions}

Characterisation results were compared with those resulting from the Doehlert experimental design methodology and revealed (i) an optimisation of thermal stability and tensile mechanical properties via the parametric printing process depending on the formulation, and (ii) lower viscosities due to a pre-degradation of the polymer matrix with the addition of OMLS during compounding and to a greater dependency of viscosity on temperature with semi-diluted systems, accompanied by a lower nozzle clogging probability and so a lower failure rate and a better printability for PLA charged at intermediate loadings. The presented methodology allows a deeper mastery of the manufacturing chain by simultaneously controlling materials formulation and process-induced structure to freely tailor thermomechanical performance of additively manufactured composites. Numerical modelling based on this experimental method would help design parts by predicting multi-physics characteristics, which would reduce the number of test-failure iterations in applications where waste must be limited, such as, for example, in space station with on-demand tooling or repairing.

Several recommendations are presented in this work. Despite an improved fracture toughness with printed nanocomposites compared to injected moulded nanocomposites, future studies should focus on new formulations with plasticiser since PLA remains a brittle matrix. As another way, formulations with nanohydroxyapatite could help design functional scaffolds for bone tissue engineering applications in combination with barrier properties of MMT for drug-controlled release. Future studies should also focus on the filament ageing. As MMT is a nucleating agent and the filaments are amorphous, a crystallinity could appear on the long term and cause the filament to gain brittleness. Other additives should be sought to enhance the viability of functional nanocomposites with FFF.

\section{Acknowledgements}

The project PolyFabAdd is co-funded by the European Union. Europe invests in Champagne-Ardenne with the European Regional Development Fund. The authors gratefully acknowledge the Région Champagne-Ardenne (France) (grant number D201501201) and the European Union for their financial support (grant number D201505025). This project is also included in the MATUR Chair, co-financed by the Région Champagne-Ardenne (France) (grant number D201507798), the European Union (grant number D201507799) and UIMM (Partners convention n $13-2015$ ). Dr. Florica Lazar and Mr. Stéphane Buet are thanked for their technical support in this project.

\section{References}

[1] Ngo TD, Kashani A, Imbalzano G, Nguyen KTQ, Hui D. Additive manufacturing (3D printing): A review of materials, methods, applications and challenges. Compos Part B Eng 2018;143:172-96. https://doi.org/10.1016/j.compositesb.2018.02.012.

[2] Bekas DG, Hou Y, Liu Y, Panesar A. 3D printing to enable multifunctionality in polymer-based composites: A review. Compos Part B Eng 2019;179:107540. https://doi.org/10.1016/j.compositesb.2019.107540.

[3] Wang X, Jiang M, Zhou Z, Gou J, Hui D. 3D printing of polymer matrix composites: A review and prospective. Compos Part B Eng 2017;110:442-58. https://doi.org/10.1016/j.compositesb.2016.11.034.

[4] de Leon AC, Chen Q, Palaganas NB, Palaganas JO, Manapat J, Advincula RC. High performance polymer nanocomposites for additive manufacturing applications. React Funct Polym 2016;103:141-55. https://doi.org/10.1016/j.reactfunctpolym.2016.04.010.

[5] Campbell TA, Ivanova OS. 3D printing of multifunctional nanocomposites. Nano Today 2013;8:119-20. https://doi.org/10.1016/j.nantod.2012.12.002.

[6] Hassan A, Balakrishnan H, Akbari A. Polylactic Acid Based Blends, Composites and Nanocomposites. In: Thomas S, Visakh PM, Mathew AjiP, editors. Adv. Nat. Polym., vol. 18, Berlin, Heidelberg: Springer Berlin Heidelberg; 2013, p. 361-96. https://doi.org/10.1007/978-3-642-20940-6_11.

[7] Sinha Ray S, Yamada K, Okamoto M, Fujimoto Y, Ogami A, Ueda K. New polylactide/layered silicate nanocomposites. 5. Designing of materials with desired properties. Polymer 2003;44:6633-46. https://doi.org/10.1016/j.polymer.2003.08.021.

[8] Ivanova O, Williams C, Campbell T. Additive manufacturing (AM) and nanotechnology: promises and challenges. Rapid Prototyp J 2013;19:353-64. https://doi.org/10.1108/RPJ-12-2011-0127. 
[9] Armentano I, Bitinis N, Fortunati E, Mattioli S, Rescignano N, Verdejo R, et al. Multifunctional nanostructured PLA materials for packaging and tissue engineering. Prog Polym Sci 2013;38:1720-47. https://doi.org/10.1016/j.progpolymsci.2013.05.010.

[10] Mueller S, Llewellin EW, Mader HM. The rheology of suspensions of solid particles. Proc R Soc Math Phys Eng Sci 2010;466:1201-28. https://doi.org/10.1098/rspa.2009.0445.

[11] Ginoux G, Dony P, Vroman I, Alix S. Decrease in non-linear viscosity of a polylactide nanocomposite with regard to the clay volume fraction. Rheol Acta 2020. https://doi.org/10.1007/s00397-020-01199-4.

[12] Ginoux G, Vroman I, Alix S. Influence of fused filament fabrication parameters on tensile properties of polylactide/layered silicate nanocomposite using response surface methodology. J Appl Polym Sci 2021;138:14.

[13] Alix S, Follain N, Tenn N, B. A, Bourbigot S, Soulestin J, et al. Effect of highly exfoliated and oriented organoclays on the barrier properties of polyamide 6 based nanocomposites. J Phys Chem C 2012;116:4937-47. https://doi.org/doi:10.1021/jp2052344.

[14] Paspali A, Bao Y, Gawne DT, Piestert F, Reinelt S. The influence of nanostructure on the mechanical properties of 3D printed polylactide/nanoclay composites. Compos Part B Eng 2018;152:160-8. https://doi.org/10.1016/j.compositesb.2018.07.005.

[15] Li L, Sun Q, Bellehumeur C, Gu P. Composite Modeling and Analysis for Fabrication of FDM Prototypes with Locally Controlled Properties. J Manuf Process 2002;4:129-41. https://doi.org/10.1016/S1526-6125(02)70139-4.

[16] Sood AK, Ohdar RK, Mahapatra SS. Parametric appraisal of mechanical property of fused deposition modelling processed parts. Mater Des 2010;31:287-95. https://doi.org/10.1016/j.matdes.2009.06.016.

[17] Yao T, Ye J, Deng Z, Zhang K, Ma Y, Ouyang H. Tensile failure strength and separation angle of FDM 3D printing PLA material: Experimental and theoretical analyses. Compos Part B Eng 2020;188:107894. https://doi.org/10.1016/j.compositesb.2020.107894.

[18] Dong J, Huang X, Muley P, Wu T, Barekati-Goudarzi M, Tang Z, et al. Carbonized cellulose nanofibers as dielectric heat sources for microwave annealing 3D printed PLA composite. Compos Part B Eng 2020;184:107640. https://doi.org/10.1016/j.compositesb.2019.107640.

[19] Tenn N, Follain N, Soulestin J, Crétois R, Bourbigot S, Marais S. Effect of nanoclay hydration on barrier properties of PLA/montmorillonite based nanocomposites. J Phys Chem C 2013;117:12117-35.

[20] Lim L-T, Auras R, Rubino M. Processing technologies for poly(lactic acid). Prog Polym Sci 2008;33:820-52. https://doi.org/10.1016/j.progpolymsci.2008.05.004.

[21] Zaidi L, Bruzaud S, Bourmaud A, MÃ@d Ã@ric P, Kaci M, Grohens Y. Relationship between structure and rheological, mechanical and thermal properties of polylactide/Cloisite 30B nanocomposites. J Appl Polym Sci 2009:NA-NA. https://doi.org/10.1002/app.31655.

[22] Carrasco F, Santana O, Cailloux J, Maspoch MLl. Kinetics of the thermal degradation of poly(lactic acid) obtained by reactive extrusion: Influence of the addition of montmorillonite nanoparticles. Polym Test 2015;48:69-81. https://doi.org/10.1016/j.polymertesting.2015.09.014.

[23] Sinha Ray S, Yamada K, Okamoto M, Ueda K. New polylactide-layered silicate nanocomposites. 2. Concurrent improvements of material properties, biodegradability and melt rheology. Polymer 2003;44:857-66. https://doi.org/10.1016/S0032-3861(02)00818-2.

[24] Saiter A, Delpouve N, Dargent E, Oberhauser W, Conzatti L, Cicogna F, et al. Probing the chain segment mobility at the interface of semi-crystalline polylactide/clay nanocomposites. Eur Polym J 2016;78:274-89. https://doi.org/10.1016/j.eurpolymj.2016.03.040.

[25] Singh S, Ghosh AK, Maiti SN, Raha S, Gupta RK, Bhattacharya S. Morphology and rheological behavior of polylactic acid/clay nanocomposites. Polym Eng Sci 2012;52:225-32. https://doi.org/10.1002/pen.22074.

[26] Aubry T, Razafinimaro T, Médéric P. Rheological investigation of the melt state elastic and yield properties of a polyamide-12 layered silicate nanocomposite. J Rheol 2005;49:425-40. https://doi.org/10.1122/1.1859791.

[27] Vaia RA, Giannelis EP. Lattice model of polymer melt intercalation in organically-modified layered silicates. Macromolecules 1997;30:7990-9.

[28] Bordes P, Pollet E, Averous L. Nano-biocomposites: Biodegradable polyester/nanoclay systems. Prog Polym Sci 2009;34:125-55. https://doi.org/10.1016/j.progpolymsci.2008.10.002.

[29] Di Y, Iannace S, Di Maio E, Nicolais L. Poly(lactic acid)/organoclay nanocomposites: Thermal, rheological properties and foam processing. J Polym Sci Part B Polym Phys 2005;43:689-98.

[30] Vasanthakumari R, Pennings AJ. Crystallization kinetics of poly(1-lactic acid). Polymer 1983;24:175-8. https://doi.org/10.1016/0032-3861(83)90129-5.

[31] Zhang J, Tashiro K, Tsuji H, Domb AJ. Disorder-to-order phase transition and multiple melting behavior of poly(Llactide) investigated by simultaneous measurements of WAXD and DSC. Macromolecules 2008;41:1352-7.

[32] Wu D, Cheng Y, Feng S, Yao Z, Zhang M. Crystallization Behavior of Polylactide/Graphene Composites. Ind Eng Chem Res 2013;52:6731-9. https://doi.org/10.1021/ie4004199.

[33] Sarwar Z, Yousef S, Tatariants M, Krugly E, Čiužas D, Danilovas PP, et al. Fibrous PEBA-graphene nanocomposite filaments and membranes fabricated by extrusion and additive manufacturing. Eur Polym J 2019;121:109317. https://doi.org/10.1016/j.eurpolymj.2019.109317. 
[34] Lee JH, Park TG, Park HS, Lee DS, Lee YK, Yoon SC, et al. Thermal and mechanical characteristics of poly(l-lactic acid) nanocomposite scaffold. Biomaterials 2003;24:2773-8. https://doi.org/10.1016/S0142-9612(03)00080-2.

[35] Gu S-Y, Ren J, Dong B. Melt rheology of polylactide/montmorillonite nanocomposites. J Polym Sci Part B Polym Phys 2007;45:3189-96.

[36] Cho JW, Paul DR. Nylon 6 nanocomposites by melt compounding. Polymer 2001;42:1083-94. https://doi.org/10.1016/S0032-3861(00)00380-3.

[37] Krishnamachari P, Zhang J, Lou J, Yan J, Uitenham L. Biodegradable poly(lactic acid)/clay nanocomposites by melt intercalation: A study of morphological, thermal, and mechanical properties. Int J Polym Anal Charact 2009;14:33650.

[38] Lai S-M, Wu S-H, Lin G-G, Don T-M. Unusual mechanical properties of melt-blended poly(lactic acid) (PLA)/clay nanocomposites. Eur Polym J 2014;52:193-206. https://doi.org/10.1016/j.eurpolymj.2013.12.012.

[39] Odegard GM, Clancy TC, Gates TS. Modeling of the Mechanical Properties of Nanoparticle/Polymer Composites. Polymer 2005;46:553-62.

[40] Davis CS, Hillgartner KE, Han SH, Seppala JE. Mechanical strength of welding zones produced by polymer extrusion additive manufacturing. Addit Manuf 2017;16:162-6. https://doi.org/10.1016/j.addma.2017.06.006.

[41] Bellehumeur C, Li L, Sun Q, Gu P. Modeling of Bond Formation Between Polymer Filaments in the Fused Deposition Modeling Process. J Manuf Process 2004;6:170-8. https://doi.org/10.1016/S1526-6125(04)70071-7. 